\title{
Catalyst Halogenation Enables Rapid and Efficient Polymerizations with Visible to Near-Infrared Light
}

\author{
Alex Stafford, ${ }^{a}$ Dowon Ahn, ${ }^{a}$ Emily K. Raulerson, ${ }^{a}$ Kun-You Chung, ${ }^{a}$ Kaihong Sun, ${ }^{a}$ Danielle M. \\ Cadena, ${ }^{a}$ Elena M. Forrister, ${ }^{b}$ Shane R. Yost,${ }^{b}$ Sean T. Roberts, ${ }^{a}$ and Zachariah A. Page ${ }^{* a}$ \\ ${ }^{a}$ Department of Chemistry, The University of Texas at Austin, Austin, Texas 78712, USA.

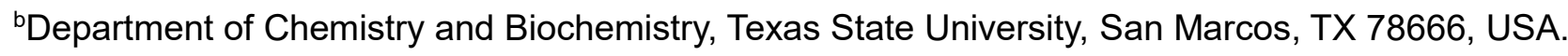 \\ *email: zpage@utexas.edu
}

\section{Abstract}

Driving rapid polymerizations with visible to near-infrared (NIR) light will enable nascent technologies in the emerging fields of bio- and composite-printing. However, current photopolymerization strategies are limited by long reaction times, high light intensities, and/or large catalyst loadings. Improving efficiency remains elusive without a comprehensive, mechanistic evaluation of photocatalysis to better understand how composition relates to polymerization metrics. With this objective in mind, a series of methine- and aza-bridged boron dipyrromethene (BODIPY) derivatives were synthesized and systematically characterized to elucidate key structure-property relationships that facilitate efficient photopolymerization driven by visible to NIR light. For both BODIPY scaffolds, halogenation was shown as a general method to increase polymerization rate, quantitatively characterized using a custom real-time infrared spectroscopy setup. Furthermore, a combination of steady-state emission quenching experiments, electronic structure calculations, and ultrafast transient absorption revealed that efficient intersystem crossing to the lowest excited triplet state upon halogenation was a key mechanistic step to achieving rapid photopolymerization reactions. Unprecedented polymerization rates were achieved with extremely low light intensities $\left(<1 \mathrm{~mW} / \mathrm{cm}^{2}\right)$ and catalyst loadings $(<50 \mu \mathrm{M})$, exemplified by reaction completion within 60 seconds of irradiation using green, red, and NIR light-emitting diodes.

\section{Introduction}

Light has been used to rapidly convert liquid resins (i.e., photopolymers) into solid objects in a process known as photocuring. This process provides control over when and where a reaction occurs (i.e., spatiotemporal control) and has enabled transformative technological advancements in imaging, 
photolithography, adhesives, coatings, and, most recently, stereolithographic 3D printing. ${ }^{1-7}$ In particular, enormous growth of the field came at the advent of photolithography, which today has been widely adopted to manufacture modern microelectronics, such as microprocessors and memory chips, ubiquitous in computers, phones, and cars. ${ }^{8-11}$ In contemporary photolithography, high energy UV light $(<400 \mathrm{~nm})$ is used to prepare 2D polymer patterns on surfaces. Facilitated by the digital revolution, lessons learned from 2D photopatterning have been effectively extended to the rapid preparation of $3 \mathrm{D}$ objects with customized form factors via additive manufacturing (i.e., 3D printing). Operating by successive photocuring of individual layers, stereolithography takes a computer-aided design from the screen to our hands. ${ }^{3}$ As with photolithography, current stereolithographic techniques rely on UV/violet light $(<420 \mathrm{~nm})$ for photocuring, which limits material compatibility due to degradation and attenuation by absorption or scattering of high energy photons.

The recent widespread availability of inexpensive visible light emitting diodes (LEDs) offers an alternative method to UV photocuring that provides 1) milder and more cost-effective reaction conditions, 2) larger penetration depths due to reduced scattering and background absorption, and 3) wavelengthselectivity (i.e., the ability to activate different chemical pathways with individual wavelengths/colors of electromagnetic radiation). ${ }^{12-17}$ As such, visible light photocuring holds the potential to promote the preparation of, inter alia, biocompatible materials containing UV-absorbing or -sensitive components, strong and lightweight composite structures, and multimaterial objects having predefined functionality embedded in discrete domains. For example, in dentistry photocurable coatings have shifted to blue irradiation to mitigate risks associated with UV exposure by employing visible light absorbing photocatalysts (e.g., camphorquinone and acylgermanes).18,19 However, photocuring with longer wavelengths of light (green to near infrared, NIR) is an ongoing challenge that, to date, has been restricted to long exposure times (> $60 \mathrm{~s}$ ) and/or high intensity irradiation $\left(>50 \mathrm{~mW} / \mathrm{cm}^{2}\right)$, precluding their utility in photocuring applications. ${ }^{12-17}$

To address the grand challenge of efficient photocuring with visible to NIR light, a number of metal and metal-free photocatalysts have been examined for their ability to induce polymerization. ${ }^{12,16}$ These photocatalysts fall into one of two categories: Type I or Type II. Type I photosystems consist only of a photoinitiator $(\mathrm{PI})$ that degrades upon light absorption to yield reactive fragments capable of initiating 
polymerization (e.g., radicals, cations, or anions). Type II systems on the other hand, require at least two components, an initiator and either a hydrogen donor or a photosensitizer (PS), to produce reactive compounds capable of initiating polymerization. While Type I systems rely on a "forbidden" $n \rightarrow \pi^{*}$ transition, Type II systems operate through $\Pi \rightarrow \pi^{*}$ excitation with characteristically stronger molar absorptivity at longer wavelengths of light (> $500 \mathrm{~nm}$, green to NIR). Attractive traits accompanying Type II systems that activate with low-energy light include being environmentally benign, having minimal side reactions, and not releasing volatile organic compounds. ${ }^{20,21}$ However, reactions are often slower for Type II systems in comparison to Type I due to a rate-limiting intermediate atom- or electron-transfer step that relies on an effective collision between an initiator and excited PS to ultimately produce the requisite reactive units. Therefore, we hypothesize that efficiency of a Type II photosystem can be improved by increasing the excited state lifetime of a PS, which in-turn will increase the number of initiator collisions that occur per photon absorbed.

Xanthenes and cyanines have received considerable attention as Type II PS compounds, with demonstrated photocuring under visible and NIR light, respectively. ${ }^{20-22}$ Both Lalevée ${ }^{16,23,24}$ and Strehmel ${ }^{25-27}$ and their coworkers have elegantly shown visible-to-NIR photocuring of acrylates and epoxies using Type II photosystems. For example, recent reports have shown that NIR polymerization chemistry can be either rapid (<60 s) when using a high intensity $\left(\geq 400 \mathrm{~mW} / \mathrm{cm}^{2}\right)$ laser diode at 785 $\mathrm{nm},{ }^{23}$ or reactive under low intensity $\left(\sim 30 \mathrm{~mW} / \mathrm{cm}^{2}\right)$ LED light centered at $\sim 790 \mathrm{~nm}$, yet requiring longer exposure times $(>100 \mathrm{~s})^{25}$. This apparent tradeoff between reaction rate and incident light intensity necessitates a closer examination of visible-to-NIR photosystems to advance state-of-the-art photocuring. To this end, an opportunity remains to simultaneously examine critical facets of photocuring by: 1) quantifying apparent polymerization rate under normalized irradiation conditions and 2) identifying a modular synthetic photocatalyst to facilitate a systematic structure-property study.

A series of boron-dipyrromethene (BODIPY) dyes were selected to address these photopolymerization challenges. Despite the attractive traits of BODIPYs, including tunable optoelectronic properties, high molar extinction, and facile and modular syntheses, ${ }^{28-30}$ they have received far less attention as photopolymer catalysts $24,31,32$ in comparison to their xanthene and cyanine counterparts (Scheme 1). However, the BODIPY platform has been heavily examined for imaging, ${ }^{33,34}$ sensing, ${ }^{35}$ and photodynamic 
therapy ${ }^{36,37}$ applications. These extensive studies have unveiled a remarkable range of optoelectronic properties for compounds bearing a BODIPY scaffold, showing peak absorption values from $500-900 \mathrm{~nm}$ (blue/green-NIR), extinction coefficients exceeding $5 \times 10^{4} \mathrm{M}^{-1} \mathrm{~cm}^{-2}$, and reduction and oxidation potentials that span from -2.40 to $-0.35 \mathrm{~V}$ and 1.55 to $0.18 \mathrm{~V}$ (vs. $\mathrm{Cp}_{2} \mathrm{Fe}_{\mathrm{Cp}} \mathrm{Fe}+$ ), respectively. ${ }^{28-30}$ Most important for the present application is efficient intersystem crossing (ISC) to long-lived triplet excited states that has been observed for various BODIPYs. Previously, these triplet excited states have played an important role in photodynamic therapy by generating reactive singlet oxygen, ${ }^{38}$ and are presented here for the first time in photocuring applications. A common method to increase the ISC rate in BODIPYs has been halogenation, which relies on the so called "heavy atom effect" 39 where spin-orbit coupling is enhanced through incorporation of elements with high atomic number. This impressive versatility in absorption, redox potentials, and excited state dynamics make BODIPY dyes ideal candidates for visible to NIR photocuring.

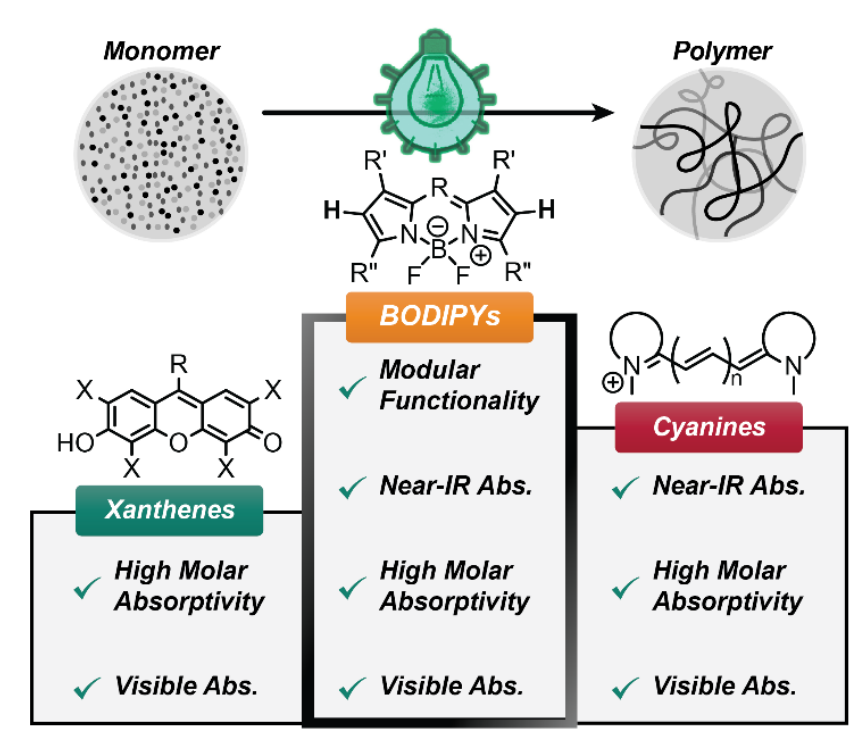

Scheme 1. Boron dipyrromethene (BODIPY) as an attractive photocatalyst platform for visible to NIR photopolymerizations, in place of traditional xanthene and cyanine derivatives.

Herein, a small library of BODIPYs are synthesized and quantitatively examined using a custom FTIR setup $^{40}$ to systematically unveil design parameters that will serve as a guide to advance photopolymer development and associated technologies. Specifically, halogenation is comprehensively examined as a universal method to improve photocuring efficiency, which builds off the hypothesis that longer lived excited states will increase the probability of a successful collision in a Type II initiation process. Triplet 
state formation is shown to be critical as photocatalyst efficiency increases with ISC rates. PS halogenation results in a 5-8x rate increase for different BODIPY classes, and demonstrates unprecedented photopolymerization sensitivity at low PS loadings (down to $0.001 \mathrm{~mol} \%=47 \mu \mathrm{M}$ ) and light intensities for green $\left(\sim 0.01 \mathrm{~mW} / \mathrm{cm}^{2}\right)$ and NIR $\left(\sim 1 \mathrm{~mW} / \mathrm{cm}^{2}\right)$ exposure. Thus, halogenation to form long-lived excited states is shown to be a general method to effectively increase photopolymerization efficiency, which provides a platform to enable emerging photocuring applications.

\section{Results and Discussion}

A set of BODIPY dyes were synthesized to contain a mesityl group at the bridgehead position along with 4 methyl substitutions on the periphery, providing both solubility and stability against nucleophilic attack. ${ }^{41}$ As shown in Figure 1a, the synthesis of the hydrogen-functionalized derivative (Mes-H) was accomplished via reductive coupling between commercially available 2,4-dimethylpyrrole and mesitaldehdye in a facile one pot approach. ${ }^{42}$ The resulting modular Mes-H derivative served as a framework to study the heavy atom effect with respect to photopolymer catalysis. Subsequent halogenation of Mes-H with the respective $\mathrm{N}$-halosuccinimide derivative yielded the desired chloro(Mes-Cl), bromo- (Mes-Br), and iodo- (Mes-I) substituted BODIPY dyes. Upon isolation it was immediately apparent by eye that each compound had a distinct color in solution, ranging from green to deep red (Figure 1b, inset).

To quantitatively compare the photocatalytic activity between different BODIPY derivatives it was critical to first characterize their optical properties (e.g., absorption profiles and corresponding extinction coefficients). UV-vis absorption spectroscopy in dilute solution revealed that halogenation resulted in a $\sim 30 \mathrm{~nm}$ red-shift of the peak absorption wavelength $\left(\lambda_{\max }\right)$ (Figure 1b). Specifically, the $\lambda_{\max }$ values were 497, 523, 523, and 529 nm for Mes-H. - Cl, - Br, and -I derivatives, respectively (Table 1). Extinction coefficients were determined using Beer's law with a calibration curve generated from solutions containing different concentrations of each BODIPY derivative. As is typical for BODIPY's, the extinction coefficients at $\lambda_{\max }$ were quite high, ranging from $\sim 67,000-86,000 \mathrm{M}^{-1} \mathrm{~cm}^{-1}$ (Table 1). The strong absorption cross section with respect to the green LED, shown in Figure 1b (and Figure S9 in the SI), 
for the present derivatives is beneficial for photopolymers as it can facilitate the use of lower catalyst loadings and/or light intensities.

After isolating the four BODIPY derivatives their utility as photosensitizers to induce polymerization was evaluated. The polymerizations were carried out using neat isobornyl acrylate as the monomer, selected for its low volatility and commercial viability. As a testament to the versatility of the present photosystem, 2-hydroxy ethyl acrylate and $\mathrm{N}, \mathrm{N}$-dimethyl acrylamide were also examined and provided similar photopolymerization results to those described below with isobornyl acrylate (Figures S19 and $\mathbf{S 2 0}$ in the SI). The initiator concentrations used were akin to those reported by Lalevée and coworkers, ${ }^{32}$ however reported as mol\% in lieu of wt\% for direct comparison. Specifically, $0.1 \mathrm{~mol} \%$ of the BODIPY photosensitizer, 0.1 mol\% of the donor (D) initiator 2-(butyryloxy)- $N, N, N$-trimethylethan-1-aminium butyltriphenylborate, and $1.0 \mathrm{~mol} \%$ of the acceptor (A) initiator [4-(octyloxy)phenyl](phenyl)iodonium hexafluoroantimonate diphenyliodonium, were fully dissolved in the monomer (Figure 2a). Degassing the mixture with nitrogen or argon followed by irradiation with a green LED resulted in rapid polymerization, evident by solidification in less than 60 seconds. To quantitatively characterize this (a)
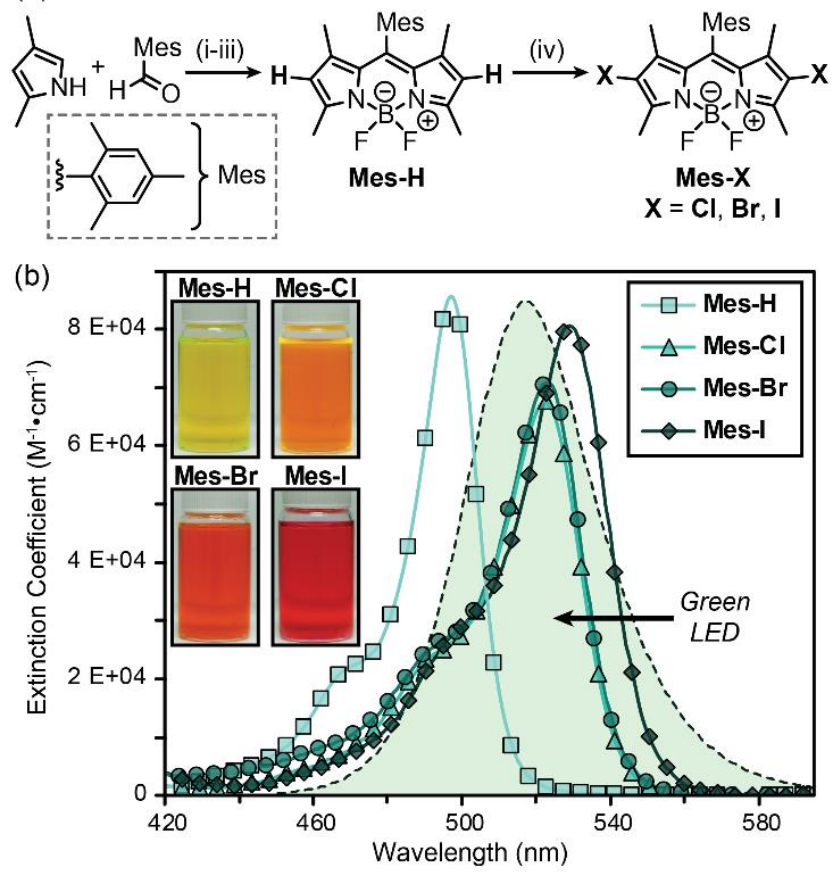

Figure 1. Mesityl-functionalized BODIPY dyes. (a) Modular synthetic procedure to four different derivatives. (b) UV-vis absorption spectroscopy $(5-10 \mu \mathrm{M}$ in acetonitrile). Normalized green LED emission profile overlaid with the UV-vis absorption spectra in (b), showing near full spectral overlap with the halogenated derivatives and partial overlap with Mes-H. Inset - photographs of distinctly colored BODIPYs in solution. Reagents and conditions: (i) TFA, DCM, 3hrs.; (ii) DDQ, 1hr.; (iii) $\mathrm{NEt}_{3}, \mathrm{BF}_{3} \cdot \mathrm{OEt}_{2}$, 2hrs. 65\% (two steps); (iv) NCS, DCM (58\%) (X=Cl); NBS, DCM (50\%) (X=Br); NIS, DCM:MeOH (65\%) (X=I) 
polymerization process, a novel method to monitor reaction kinetics was developed using FTIR spectroscopy ${ }^{40}$ A schematic representation of the attenuated total reflectance (ATR) FTIR setup is shown in Figure $\mathbf{2 b}$. Critically, LED and IR irradiation occur from the same face of the ATR crystal (bottom-up). This setup enables precise kinetics data to be rapidly collected for opaque photochemical transformations by providing uniform irradiation and shallow sample measurements. Within the Mid-IR region the depth of measurement ranges from $\sim 0.5-5 \mu \mathrm{m}$, depending on the spectral position of the absorption signal (see "normalizing photons absorbed" in the SI for more details). For acrylic polymerizations, the disappearance of the $\mathrm{C}=\mathrm{C}$ vinylic stretch at $808 \mathrm{~cm}^{-1}$ was monitored and used to determine percent double bond conversion, ${ }^{43}$ corresponding to a sampling depth of $2.5 \mu \mathrm{m}$. Simple glassware was made to encase the sample and purge its surrounding atmosphere with inert gas (e.g., nitrogen) to eliminate oxygen inhibition of the radical polymerization. To directly compare photocuring efficiency between different BODIPY dyes with distinct absorption profiles, the number of photons absorbed by each sample was normalized. This was accomplished by varying the photon flux from a calibrated green LED after integrating the area of overlap between the absorption profile for each BODIPY derivative with the emission profile of the LED (Figure 1c) (see SI for more details). Notably, Mes-H has the smallest absorption cross section with the green LED emission compared to the halogenated BODIPY's, which was compensated by using a 2.2-2.3 times higher photon flux (i.e., light intensity) to equate the number of photons absorbed.

Photopolymerization kinetics were monitored using real time ATR-FTIR to determine the photocatalytic efficiency across the BODIPY series (Figure 2c). A dramatic enhancement in the rate of polymerization was observed $(>2 x)$ when employing halogenated vs non-halogenated derivatives (Table 1). Furthermore, a trend in the halogenated series was detected, with increasing maximum polymerization rates $(0.43 \pm 0.03$ to $0.91 \pm 0.02$ to $0.95 \pm 0.03 \mathrm{M} / \mathrm{s})$ correlating to an increase in the atomic number of the heavy atom; $-\mathbf{C l}$ to $-\mathrm{Br}$ to $-\mathbf{I}$, respectively. As a negative control, no polymerization was observed within the first $10 \mathrm{~s}$ prior to light exposure, indicative of resin stability in the dark and that light is necessary to drive the polymerization. Moreover, resins stored in the dark for $\sim 3$ days showed no observable loss in activity. Upon turning the light on, all polymerizations rapidly initiated and reached a maximum monomer-to-polymer conversion of $\sim 80 \%$. The $\sim 80 \%$ conversion is hypothesized to be an 
upper limit for this part icular mixture due to solidification of poly(isobornyl acrylate), a glassy polymer $\left(T_{g} \approx 94^{\circ} \mathrm{C}\right)^{44}$ that "locks" remaining small molecules into place. For Mes-Br and Mes-I, the time to reach maximum conversion after turning the light on was $8.5 \pm 0.9 \mathrm{~s}$ and $6.5 \pm 0.3 \mathrm{~s}$, respectively. Impressively, this was achieved under very low light intensity, $<0.5 \mathrm{~mW} / \mathrm{cm}^{2}$, which, for comparison, is $\sim 2$ orders of magnitude less intense than an unfocused commercial laser pointer $\left(\sim 10-100 \mathrm{~mW} / \mathrm{cm}^{2}\right)$. The ability to react under low light intensity saves energy for photocuring applications and can enable 3D printing via digital light processing (DLP), where irradiation at the build plane is typically $<20 \mathrm{~mW} / \mathrm{cm}^{2}$.

Fluorescence spectroscopy was used to probe the stead-state photophysics of the Mes-BODIPY derivatives and better understand the mechanism(s) that lead to faster polymerization rates. The fluorescence quantum yield (QY) was determined following literature precedent ${ }^{45}$ in acetonitrile with

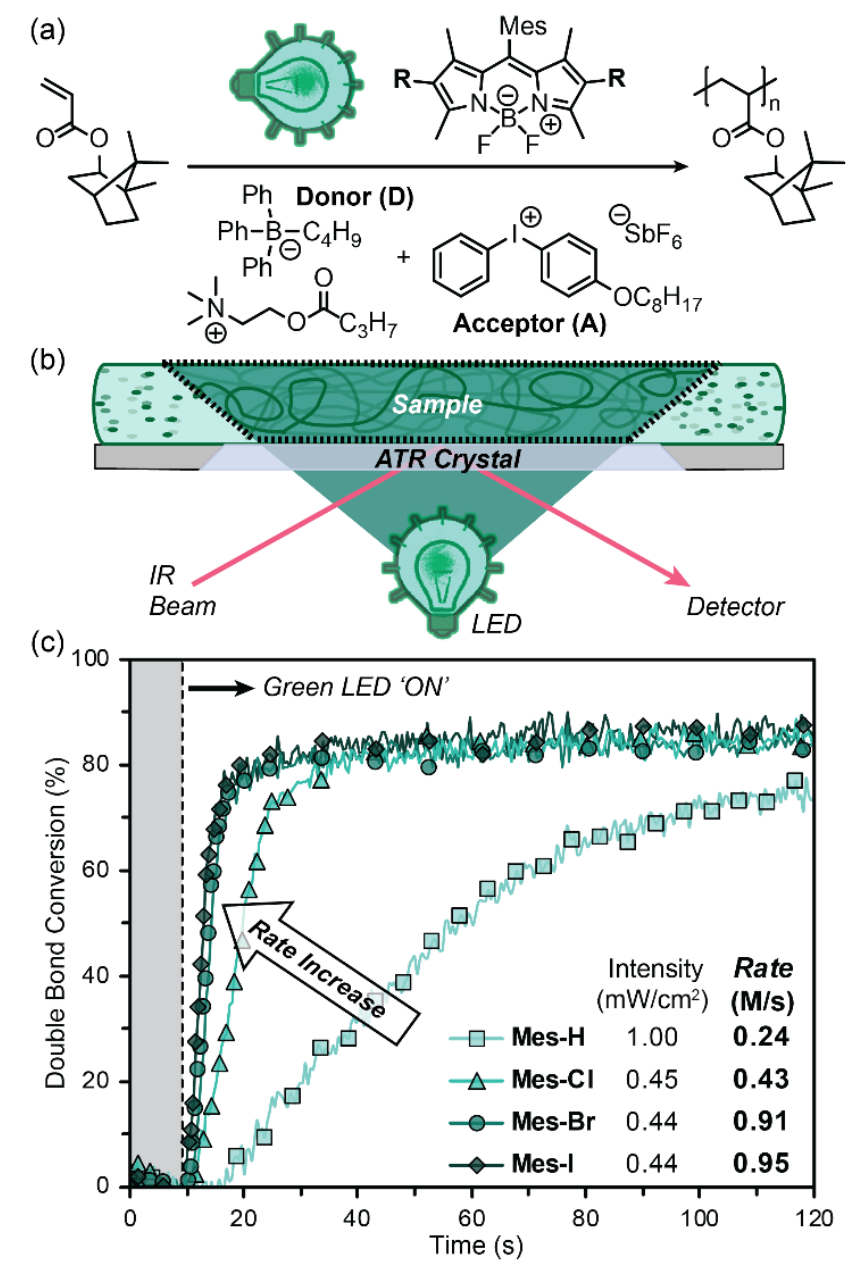

Figure 2. Photopolymerization kinetics to compare the efficiency of different Mes-BODIPY photosensitizers. (a) Chemical structures for the different components within the resin formulation: isobornyl acrylate as monomer, butyltriphenylborate salt as donor, and diphenyl iodonium as acceptor. (b) Schematic illustration for the custom real time ATR-FTIR setup with bottom-up irradiation. (c) Plot of conversion vs time using an intensity that normalizes photons absorbed between different BODIPY derivatives. No polymerization is observed in the dark (gray region). Upon irradiation polymerization rates increase in the order of Mes-H, $-\mathbf{C l},-\mathbf{B r}$, and $-\mathbf{I}$, respectively. 
Rhodamine $6 \mathrm{G}$ as a reference dye (Figure $\mathbf{3 a}$ and Figure $\mathbf{S 2 1}$ in the $\mathrm{SI}$ ). The fluorescence QY valueswere inversely related to the polymerization rate, specifically $0.94 \pm 0.06,0.70 \pm 0.05,0.19 \pm 0.02$, and $0.03 \pm 0.01$ for Mes-H, $-\mathbf{C l},-\mathbf{B r}$, and -I derivatives, respectively (Table 1). The decreased $Q Y$ for halogenated derivatives in dilute solution suggests that an intramolecular non-radiative quenching pathway outcompetes fluorescence, such as ISC.

Subsequently, intermolecular fluorescence (i.e., singlet) quenching by $\mathbf{D}$ and $\mathbf{A}$ initiators was probed to elucidate the mechanisms for radical generation (Figure 3b). The Stern-Volmer plot for Mes-H and MesBr shows quenching by both $\mathbf{D}$ and $\mathbf{A}$ compounds, represented as an increase in the ratio between initial fluorescence intensity $\left(I_{0}\right)$ and intensity $(\mathrm{I})$ at a particular concentration of $\mathbf{D}$ or $\mathbf{A}(\mathrm{i} . \mathrm{e} ., \mathrm{I} / \mathrm{l} / \mathrm{l})$. This suggests that some contribution towards polymerization derives from the singlet photoexcited state for both Mes$\mathbf{H}$ and Mes-Br, although to a lesser extent for Mes-Br as represented by the weaker quenching. Consecutive additions of $\mathbf{D}$ results in a steeper slope relative to equimolar additions of $\mathbf{A}$ for both MesBODIPY derivatives. This indicates that photoexcited Mes-BODIPY dyes more readily accept an electron from $\mathbf{D}$, as opposed to donate an electron to $\mathbf{A}$. Thus, the major mechanistic pathway for this photosystem is thought to follow reductive quenching, which would result in a radical anion of the respective MesBODIPY and a butyl radical ${ }^{46}$ from the oxidized triphenylalkyl borate salt, D (Figure 3c). Importantly, while the Mes-Br results in faster polymerization rates, the Stern-Volmer plot suggests that Mes-H reacts faster with both $\mathbf{D}$ and $\mathbf{A}$, suggesting a secondary pathway is contributing to polymerization for $\mathbf{M e s}-\mathbf{B r}$. Prior to investigating the secondary pathway, cyclic voltammetry $(\mathrm{CV})$ and computational studies were performed to probe the electronic energy states of the different photosystem components to corroborate the mechanistic hypothesis that reductive quenching is favored over oxidative quenching. CV data was collected to determine the highest occupied molecular orbital (HOMO) and lowest unoccupied molecular orbital (LUMO) energy levels, as provided in Table 1 (Figures S22-29 in the SI). Halogenation stabilized both the LUMO and HOMO energy levels, although to a greater extent for the LUMO ( 0.3 eV decrease vs. $\lesssim 0.15 \mathrm{eV}$ decrease, respectively). This shift correlates to a decrease in the electronic energy gap $\left(E_{g}=E_{\text {HOMO }}-E_{L U M O}\right)$, which is consistent with the red-shift in absorption (i.e., decrease in optical energy gap) observed previously (Figure 1). The decrease in both HOMO and LUMO energy levels in going 
from Mes-H to $\mathbf{- B r}$ increases the driving force for reductive quenching and decreases it for oxidative quenching (Figure 3c), in support of the Stern-Volmer analysis.

Additionally, density functional theory was used to support the relative electronic energy level trends observed with CV and demonstrate that computational methods may be used as a predictive tool in the future. Specifically, the def2-tzvp basis set and cam-b3lyp functional were used in an implicit solvation model to compute the HOMO and LUMO energy levels. Following literature precedent for

(a)
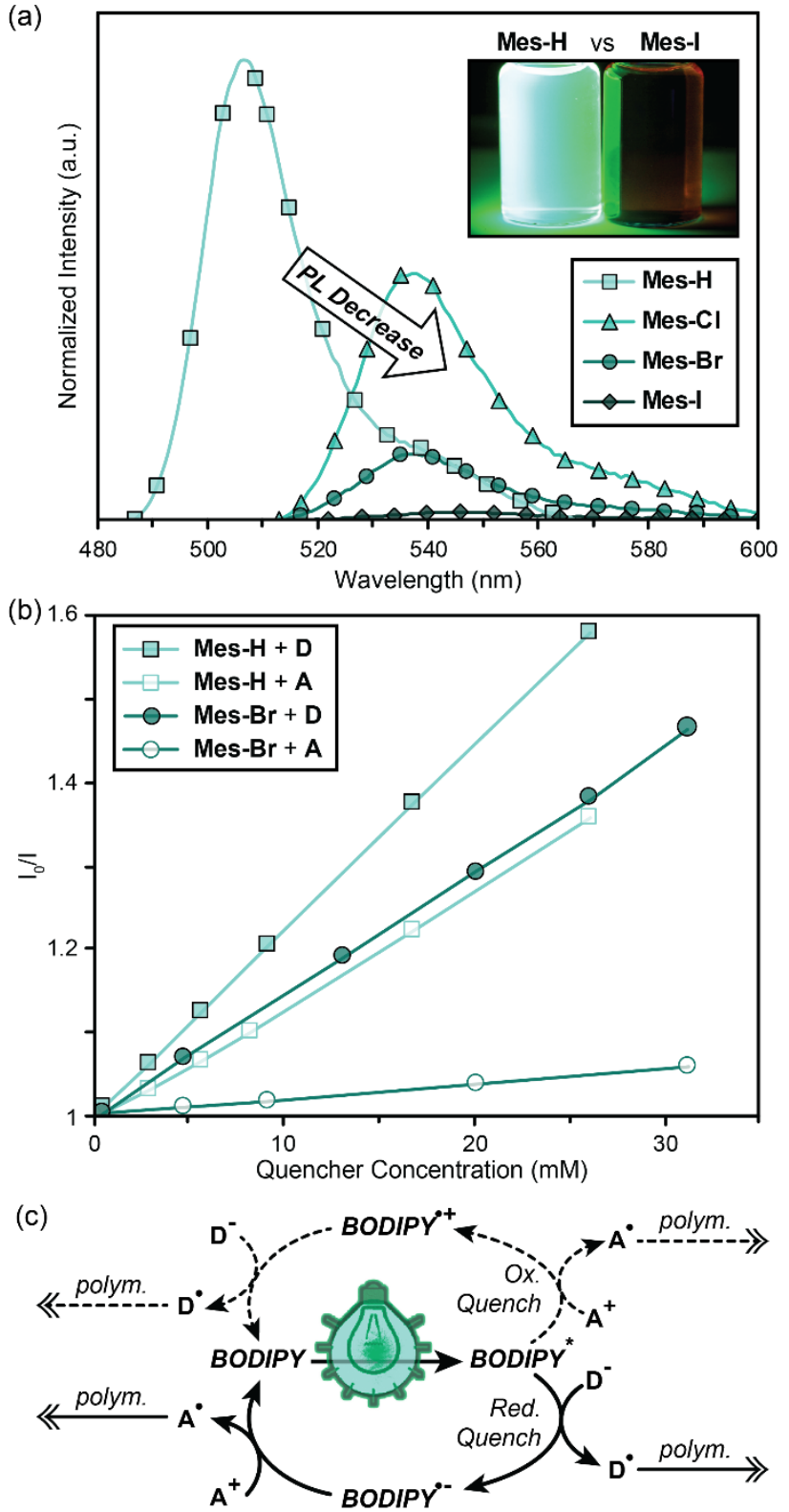

Figure 3. Fluorescence measurements in acetonitrile and proposed mechanisms for the photosystem containing MesBODIPY PS, triphenylalkylborate donor, D, and diphenyliodonium acceptor, A. (a) Photoluminescence of Mes-BODIPY series, showing a decrease in emission upon halogenation, suggestive of a non-radiative intramolecular process. Inset - photographs of Mes-H and Mes-I in acetonitrile $(0.3 \mathrm{mM})$ irradiated with a handheld $365 \mathrm{~nm}$ UV lamp. (b) SternVolmer plot of Mes-H and Mes-Br in the presence of $\mathbf{D}$ and $\mathbf{A}$, showing that $\mathbf{D}$ results in more efficient quenching for both dyes. (c) Proposed photosystem mechanisms with oxidative quenching, top, and reductive quenching (primary pathway), bottom. 
Table 1. Optoelectronic properties of the photosystem components

\begin{tabular}{|c|c|c|c|c|c|c|c|c|c|}
\hline BODIPY & $\begin{array}{c}\lambda_{\max } \\
(\mathrm{nm})\end{array}$ & $\begin{array}{c}\varepsilon\left(\mathrm{x} 10^{4}\right. \\
\left.\mathrm{M}^{-1} \mathrm{~cm}^{-1}\right)\end{array}$ & $\begin{array}{c}\text { Polym. } \\
\text { Rate }(\mathrm{M} / \mathrm{s})\end{array}$ & $\begin{array}{c}\text { Quantum } \\
\text { Yield }\end{array}$ & HOMO (eV) & LUMO (eV) ${ }^{\dagger}$ & $\begin{array}{c}\tau \text { Isc } \\
(\mathrm{ns})\end{array}$ & $\begin{array}{c}\text { Triplet } \\
\text { Yield }\end{array}$ & $\begin{array}{c}\text { Triplet Life- } \\
\text { time }(\mathrm{ns})\end{array}$ \\
\hline Mes-H & 497 & $8.6 \pm 0.2$ & $0.24 \pm 0.02$ & $0.94 \pm 0.06$ & $-5.64(-5.42)$ & $-3.16(-3.06)$ & - & - & - \\
\hline Mes-CI & 523 & $6.7 \pm 0.2$ & $0.43 \pm 0.03$ & $0.70 \pm 0.05$ & $-5.81(-5.59)$ & $-3.41(-3.25)$ & 7.8 & 0.17 & 370 \\
\hline Mes-Br & 523 & $6.9 \pm 0.3$ & $0.91 \pm 0.02$ & $0.19 \pm 0.02$ & $-5.81(-5.60)$ & $-3.41(-3.23)$ & 2.9 & 0.35 & 370 \\
\hline Mes-I & 529 & $7.9 \pm 0.3$ & $0.95 \pm 0.03$ & $0.03 \pm 0.01$ & $-5.82(-5.54)$ & $-3.37(-3.29)$ & 0.2 & 0.89 & 370 \\
\hline
\end{tabular}

*Donor HOMO $\approx-5.14 \mathrm{eV}$; Acceptor LUMO $\approx-3.51 \mathrm{eV}$ determined with cyclic voltammetry

†Values in parentheses are computed energy levels using a def2-tzvp basis set and cam-b3lyp functional

diphenyliodinium, ${ }^{47,48}$ accurate estimations for the $\mathbf{D}$ and $\mathbf{A}$ initiators were achieved by using a concerted ionization and bond cleaving process (see "computational methods/data" in the SI for more details). As shown in Table 1, the computational values for the HOMO and LUMO energies are consistently within $\sim 0.2 \mathrm{eV}$ of experiment. Both $\mathrm{CV}$ and computation confirm that there is a larger energetic driving force for reductive over oxidative quenching in the present photosystems, substantiating the preference for electron transfer from D to photoexcited Mes-BODIPY. Thus far, optoelectronic characterization has focused on the lowest excited singlet $\left(S_{1}\right)$ state of the Mes-BODIPY dyes, but fluorescence quenching experiments suggest that halogenation provides an additional mechanism to facilitate higher polymerization rates.

Transient absorption (TA) spectroscopy was employed to better assess the connection between MesBODIPY excited state dynamics and photocatalytic efficiency. As a representative, Figure 4a plots TA spectra of Mes-I dissolved in acetonitrile following photoexcitation at $530 \mathrm{~nm}$ (Mes-H, Mes-Cl, and MesBr spectra appear in the SI, Figure S31). Upon photoexcitation of Mes-I, a broad negative signal appears from 480 to $540 \mathrm{~nm}$ that corresponds to a bleaching of its ground state absorption while stimulated emission can be seen at longer probe wavelengths that tracks the shape of Mes-l's steady-state emission spectrum. Together, these features indicate excitation of Mes-I to its $S_{1}$ state. Over time, the stimulated emission is gradually replaced by a weak induced absorption band while the ground state bleach shows little evolution, signaling conversion of the $S_{1}$ state to a new excited state. Concomitant with this change is growth of an induced absorption band centered at $440 \mathrm{~nm}$ attributed to the Mes-l's lowest excited triplet $\left(\mathrm{T}_{1}\right)$ state on the basis of prior photoinduced absorption spectra of BODIPY dyes. ${ }^{49-52}$ Global target analysis of this data (See SI for details) is used to extract an ISC rate of $197 \mathrm{ps}\left(\mathrm{k}_{\mathrm{ISC}}=5.1 \times 10^{9} \mathrm{~s}^{-1}\right)$. TA spectra of Mes-Cl and Mes-Br show similar spectral evolution, albeit with lower ISC rates (Figure 4b, 
Table 1). Following ISC, the $T_{1}$ states of Mes-I, Mes-Br, and Mes-CI display similar excited state lifetimes of 370 ns. In contrast, no evidence for $T_{1}$ state formation is see for Mes-H, which returns directly from the $S_{1}$ state to its ground state over the course of a few nanoseconds.

Importantly, the comparable rates for ISC and $\mathrm{S}_{1}$ relaxation to the ground state for Mes-Br and Mes$\mathbf{C l}$ indicate these two processes compete with one another following photoexcitation. Based on the ratio of time constants measured for these processes, we estimate $35 \%$ of photoexcited $\mathbf{M e s}-\mathrm{Br}$ molecules successfully intersystem cross to their $\mathrm{T}_{1}$ state while the triplet yield is even lower for $\mathbf{M e s}-\mathbf{C l}, 17 \%$. In contrast, the fast ISC rate of Mes-I ensures that the majority of its photoexcited population (89\%) convert to its $T_{1}$ state. We ascribe the observed enhancement in photopolymerization rate in moving from Mes$\mathbf{H}$ to Mes-I to this difference in triplet production yield. As photopolymerization requires the diffusive encounter of a photoexcited Mes-BODIPY molecule and an electron donor or acceptor, the longer lifetime of the Mes-BODIPY $\mathrm{T}_{1}$ state increases the likelihood that a photoexcited molecules will encounter a suitable partner before it deactivates to the ground state. The increased triplet production yield of Mes-I
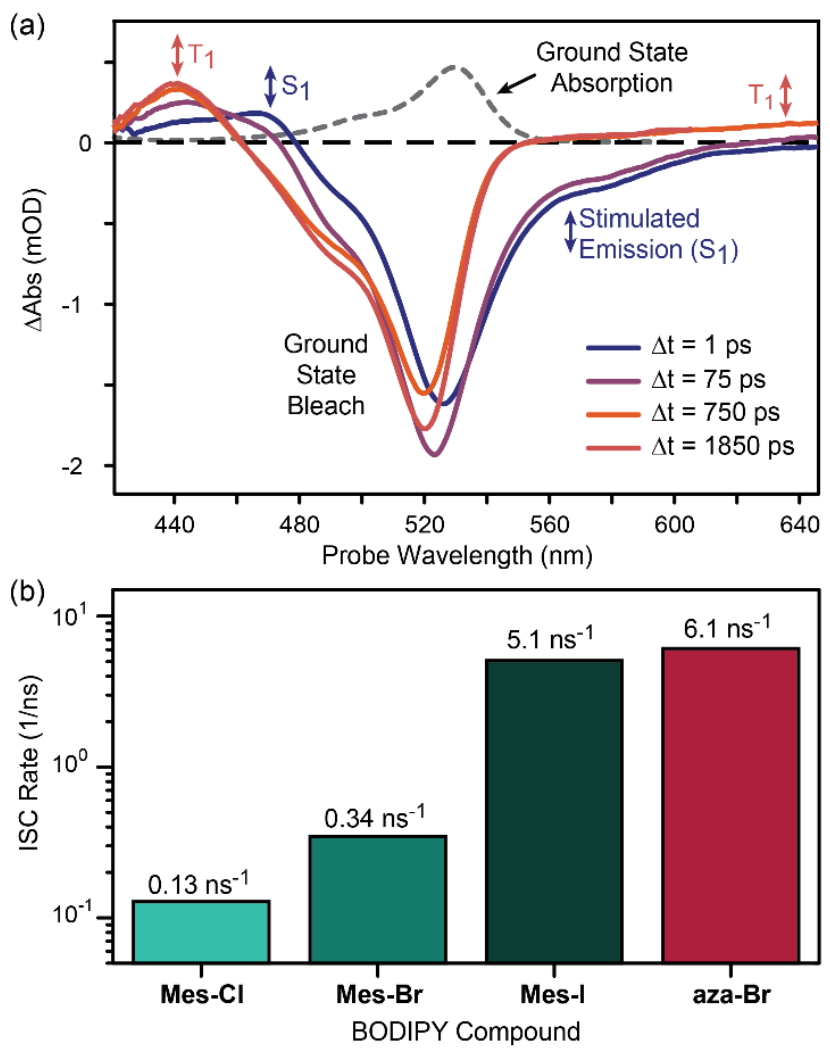

Figure 4. (a) TA spectra of Mes-I in acetonitrile, indicating that intersystem crossing occurs on a 197 ps timescale. Features tied to Mes-l's $S_{1}$ state are indicated by blue text while red text denotes features associated with Mes-l's $\mathrm{T}_{1}$ state. (b) Intersystem crossing (ISC) rates for halogenated Mes-BODIPY compounds (green) and aza-Br (red). 
over Mes- $\mathbf{C l}$ and Mes-Br ensures more photoexcitation events will result in triplet formation, thereby increasing the rate of photopolymerization.

Given the demonstrated significance of the heavy atom effect, the next aim was to develop BODIPY photocatalysts that operate effectively under irradiation from a NIR LED. To this end, the mesityl group at the meso bridgehead position was replaced with a nitrogen, and the methyl substituents off the pyrrole units with phenyl rings. ${ }^{53,54}$ Analogous to the modular Mes-BODIPY syntheses, acetophenone and benzaldehyde derivatives, as well as $\mathrm{N}$-bromosuccinimide, were employed to provide both the hydrogen, aza-H, and bromine, aza-Br, functionalized dyes (see SI for more details). As shown in Figure 5a, the combination of a nitrogen bridgehead and electron rich phenyl substituents resulted in a $\sim 135 \mathrm{~nm} \lambda_{\max }$
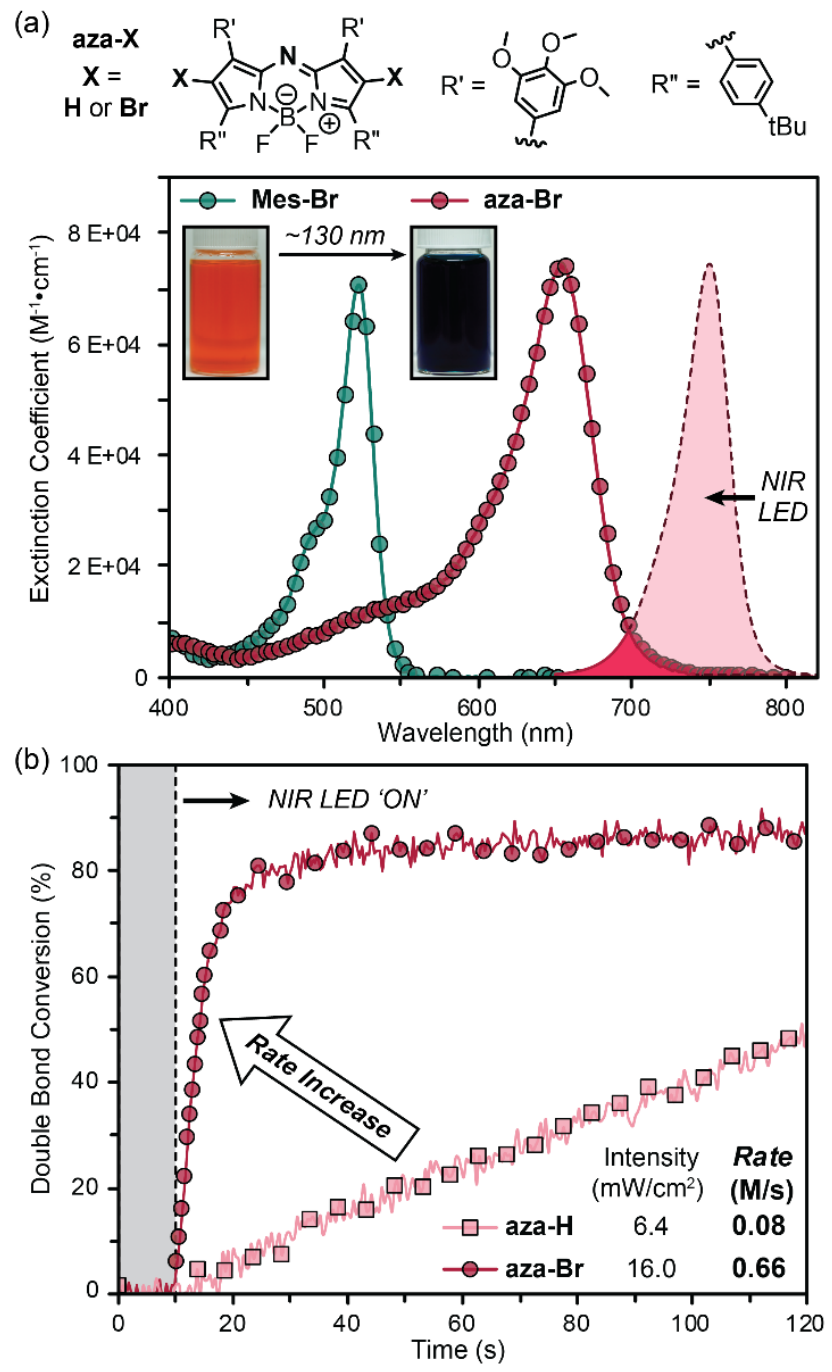

Figure 5. Photopolymerization with aza-BODIPY with a $740 \mathrm{~nm}$ NIR LED. (a) Chemical structures for aza-H and aza$\mathrm{Br}$ and UV-vis absorption spectra for Mes-Br and aza-Br (4-6 $\mu \mathrm{M}$ in acetonitrile). Inset - photographs of distinctly colored Mes-Br and aza-Br in solution. (b) Plot of conversion vs time using an irradiation intensity that normalizes photons absorbed between the two aza-BODIPY derivatives. No polymerization is observed in the dark (gray region). Upon irradiation aza-Br polymerizes at a rate $\sim 8 \times$ faster than $\mathbf{a z a}-\mathbf{H}$. 
red shift compared to the mesityl BODIPY dyes; from $~ 525 \mathrm{~nm}$ (Mes-Br) to $\sim 660 \mathrm{~nm}$ (aza-Br). Interestingly, halogenation of the aza-BODIPY resulted in a slight blue shift $(\sim 15 \mathrm{~nm})$, in contrast to that observed for the Mes-BODIPY dyes (vide supra). This blue shift requires a higher photon flux to be used in order to normalize the number of photons absorbed when exciting with a NIR LED.

Using an analogous tricomponent photosystem with aza-BODIPY as the photosensitizer in place of mesityl-BODIPY, the polymerization of isobornyl acrylate was carried out under $656 \mathrm{~nm}$ (red) and 740 nm (NIR) LEDs. The overlap between aza-BODIPY absorbance and LED emission was again used to normalize the number of photons absorbed by tuning LED intensity, providing a means to directly compare photopolymerization efficiency (Figure S9b in the SI). Monitoring the kinetics using the custom ATR-FTIR setup revealed a dramatic enhancement in polymerization rate when using aza-Br compared to aza-H, under both red (Figures S15 and S16) and NIR irradiation (Figure 5b). Under NIR light aza$\mathrm{Br}$ resulted in polymerizations that were $\sim 8 \times$ faster than aza-H $(0.66 \pm 0.03 \mathrm{M} / \mathrm{s}$ vs $0.08 \pm 0.02 \mathrm{M} / \mathrm{s})$, which corresponded to reaching maximum conversion in $11.4 \pm 0.9$ s. Such rapid NIR photopolymerization is, to the best of our knowledge, unprecedented.

Similar to Mes-BODIPY dyes, TA spectra of aza-Br and aza-H indicate bromination leads to triplet formation via ISC that is competitive with decay of the BODIPY $\mathrm{S}_{1}$ state to its ground state (Figure S33). While aza-Br undergoes ISC with a rate constant of $\mathrm{k}_{\mathrm{ISC}}=6.1 \times 10^{9} \mathrm{~s}^{-1}\left(\tau_{\mathrm{ISC}}=164 \mathrm{ps}\right)$, no appreciable triplet formation via ISC (<1\% yield) is observed for aza-H. This leads to a longer-lived triplet population upon photoexcitation of aza-Br that can initiate polymerization, while it remains absent for aza-H. Thus, we conclude that halogenation to enhance triplet formation via ISC is a general method that can improve photopolymerization efficiency.

To test the limits of photopolymerization, light intensity and catalyst loading were independently decreased for both Mes-I under green light and aza-Br under NIR light (Figure 6) until it took $\sim 60 \mathrm{~s}$ to reach maximum conversion. Impressively, using the optimized formulations a light intensity of 0.01 $\mathrm{mW} / \mathrm{cm}^{2}$ and $1 \mathrm{~mW} / \mathrm{cm}^{2}$, corresponding to an absorbed photon flux of $0.3 \times 10^{13}$ and $1.8 \times 10^{13}$ were found to be the lower bounds for green and NIR irradiation, respectively (Figure 6a). For context, the light intensity emitted by the screen of an iPhone 8 was measured to be $\sim 0.1 \mathrm{~mW} / \mathrm{cm}^{2}$ at full brightness. 
Using an intensity of $16 \mathrm{~mW} / \mathrm{cm}^{2}$ for green and NIR light, a lower limit of $0.001 \mathrm{~mol} \%(=47 \mu \mathrm{M})$ and 0.004 mol\% $(=188 \mu \mathrm{M})$ catalyst loading, corresponding to an absorbed photon flux of $4.2 \times 10^{13}$ and $1.2 \times 10^{13}$, was found for Mes-I and aza-Br, respectively (Figure 6b). Therefore, rapid photopolymerization can be achieved with extremely low irradiation intensity or catalyst loading, which is conducive to a variety of applications and ultimately will save on energy and/or material costs. Notably, the TA spectra of $\mathbf{a z a}-\mathbf{B r}$ suggests there is additional room to optimize performance of this material as deactivation of aza-Br from its $S_{1}$ state to its ground state is found to significantly compete with ISC (See SI, Figure S33). Such competition is not seen for other Mes-BODIPY dyes, suggesting modification of the functional groups that adorn aza-Br could be used to improve its ISC yield, potentially lowering the photopolymerization threshold intensity.
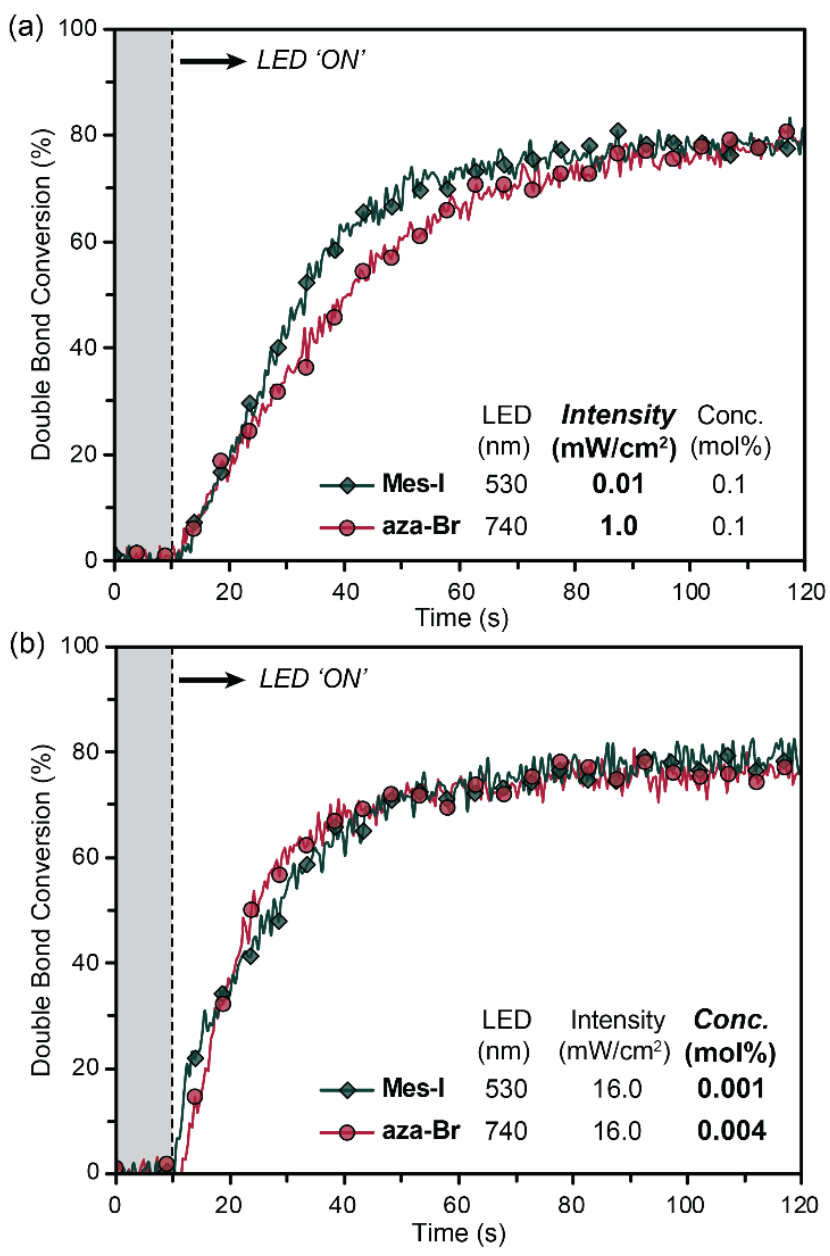

Figure 6. Photopolymerization limits for Mes-I and aza-Br under green (530 nm) and NIR (740 nm) irradiation, while maintaining near complete conversion within $\sim 60$ seconds. Minimum (a) exposure intensity and (b) photosensitizer concentration. 


\section{Concluding Remarks}

Halogenation of BODIPY dyes was demonstrated to be an effective strategy to improve the efficiency of light-driven polymerizations. Systematic kinetic and optoelectronic characterization of a series of halogenated BODIPY derivatives unveiled critical structure property insight to explain the observable efficiency enhancement. Real time ATR-FTIR with custom bottom-up irradiation was used to quantitatively characterize polymerization rate, showing that halogenation of BODIPY dyes with either mesityl or nitrogen functionality at the meso bridgehead position resulted in a 5-8× higher polymerization rate upon exposure to green, red, or NIR light. Fluorescence quenching studies suggested that electron donation into the photoexcited BODIPY dyes (i.e., reductive quenching) was the primary mechanistic pathway for radical generation. This mechanism was also supported by a larger energetic driving force identified from cyclic voltammetry and density functional theory. Transient absorption spectroscopy confirmed that the presence of "heavy atoms" (e.g., halogens) results in faster intersystem crossing rates, which corresponds to higher triplet yields and longer excited state lifetimes. In turn, the longer lifetimes improve photocatalytic efficiency by increasing the number of collisions that occur per photon absorbed between BODIPY in the excited state and an initiator. Furthermore, the halogenated BODIPY derivatives were shown to provide unprecedented photopolymerization rates (complete conversion within $\sim 60 \mathrm{~s}$ ) given extremely low catalyst loadings ( $\sim 0.001 \mathrm{~mol} \%)$ or light intensities $\left(\sim 0.01 \mathrm{~mW} / \mathrm{cm}^{2}\right.$ green light or $\sim 1$ $\mathrm{mW} / \mathrm{cm}^{2}$ NIR light). These results are particularly exciting to inform the future development of more efficient NIR photocatalysts that absorb beyond $750 \mathrm{~nm}$, which can extend the utility of photopolymers to include the formation of strong and lightweight hybrid composites and biocompatible tissue scaffolds.

\section{Conflicts of interest}

There are no conflicts to declare.

\section{Acknowledgments}

Work accomplished at the University of Texas at Austin was supported by the ARO STIR program of the Department of Defense (W911NF1910310 to Z.A.P.), Robert A. Welch Foundation (F-2007 to Z.A.P. and F-1885 to S.T.R.), and Research Corporation for Science Advancement via a Cottrell Scholars Award (24489 to S.T.R.). The authors acknowledge the use of shared research facilities supported in part by 
the Texas Materials Institute, the Center for Dynamics and Control of Materials: an NSF MRSEC (DMR1720595), and the NSF National Nanotechnology Coordinated Infrastructure (ECCS-1542159). The authors also acknowledge the Texas Advanced Computing Center (TACC) at The University of Texas at Austin for providing high performance computing resources that contributed to reported computational results. We thank Kristina Kafle, a talented undergraduate at UT Austin, for creating the TOC image.

\section{Abbreviations}

LED, light emitting diode; PI, photoinitiator; PS, photosensitizer; NIR, near infrared; UV, ultraviolet; FTIR, Fourier transform infrared; ATR, attenuated total reflectance; BODIPY, boron-dipyrromethene; Mes, mesityl; ISC, intersystem crossing; QY, quantum yield; CV, cyclic voltammetry; HOMO, highest occupied molecular orbital; LUMO, lowest unoccupied molecular orbital; TA, transient absorption; DLP, digital light processing

\section{References}

(1) Aguado, B. A.; Grim, J. C.; Rosales, A. M.; Watson-Capps, J. J.; Anseth, K. S. Engineering Precision Biomaterials for Personalized Medicine. Sci. Transl. Med 2018, 10, 8645.

(2) Camposeo, A.; Persano, L.; Farsari, M.; Pisignano, D. Additive Manufacturing: Applications and Directions in Photonics and Optoelectronics. Adv. Opt. Mater. 2019, 7 (1), 1800419.

(3) Wallin, T. J.; Pikul, J.; Shepherd, R. F. 3D Printing of Soft Robotic Systems. Nat. Rev. Mater. 2018, 3 (6), 84-100.

(4) Zhang, J.; Xiao, P. 3D Printing of Photopolymers. Polym. Chem. 2018, 9 (13), 1530-1540.

(5) Jung, K.; Corrigan, N.; Ciftci, M.; Xu, J.; Seo, S. E.; Hawker, C. J.; Boyer, C. Designing with Light: Advanced 2D, 3D, and 4D Materials. Adv. Mater. 2019, 1903850.

(6) Lim, K. S.; Galarraga, J. H.; Cui, X.; Lindberg, G. C. J.; Burdick, J. A.; Woodfield, T. B. F. Fundamentals and Applications of Photo-Cross-Linking in Bioprinting. Chem. Rev. 2020.

(7) Yu, C.; Schimelman, J.; Wang, P.; Miller, K. L.; Ma, X.; You, S.; Guan, J.; Sun, B.; Zhu, W.; Chen, S. Photopolymerizable Biomaterials and Light-Based 3D Printing Strategies for Biomedical Applications. Chem. Rev. 2020.

(8) Willson, C. G.; Dammel, R. R.; Reiser, A. Photoresist Materials: A Historical Perspective. In Advances in Resist Technology and Processing XIV; SPIE, 1997; Vol. 3049, p 28.

(9) Kozawa, T.; Santillan, J. J.; Itani, T. Relationship between Resolution Blur and Stochastic Defect of Chemically Amplified Resists Used for Extreme Ultraviolet Lithography. J. Photopolym. Sci. Technol. 2019, 32 (1), 161-167.

(10) MacDonald, S. A.; Ito, H.; Willson, C. G. Advances in the Design of Organic Resist Materials. Microelectron. Eng. 1983, 1 (4), 269-293.

(11) Ito, H.; Willson, C. G.; Frechet, J. M. J. New UV Resists with Negative or Positive Tone. In Digest of Technical Papers - Symposium on VLSI Technology; 1982; pp 86-87.

(12) Dumur, F. Recent Advances on Visible Light Metal-Based Photocatalysts for Polymerization under Low Light Intensity. Catalysts 2019, 9, 736. 
(13) Bagheri, A.; Jin, J. Photopolymerization in 3D Printing. ACS Appl. Polym. Mater. 2019, 1 (4), 593-611.

(14) Corrigan, N.; Yeow, J.; Judzewitsch, P.; Xu, J.; Boyer, C. Seeing the Light: Advancing Materials Chemistry through Photopolymerization. Angew. Chemie - Int. Ed. 2019, 58 (16), 5170-5189.

(15) Strehmel, B.; Schmitz, C.; Cremanns, K.; Göttert, J. Photochemistry with Cyanines in the Near Infrared: A Step to Chemistry 4.0 Technologies. Chem. - A Eur. J. 2019, 25 (56), 12855-12864.

(16) Xiao, P.; Zhang, J.; Dumur, F.; Tehfe, M. A.; Morlet-Savary, F.; Graff, B.; Gigmes, D.; Fouassier, J. P.; Lalevée, J. Visible Light Sensitive Photoinitiating Systems: Recent Progress in Cationic and Radical Photopolymerization Reactions under Soft Conditions. Prog. Polym. Sci. 2015, 41 (C), 32-66.

(17) Chatani, S.; Kloxin, C. J.; Bowman, C. N. The Power of Light in Polymer Science: Photochemical Processes to Manipulate Polymer Formation, Structure, and Properties. Polym. Chem. 2014, 5 (7), 2187-2201.

(18) Haas, M.; Radebner, J.; Eibel, A.; Gescheidt, G.; Stueger, H. Recent Advances in Germanium-Based Photoinitiator Chemistry. Chem. - A Eur. J. 2018, 24 (33), 8258-8267.

(19) Schroeder, W. F.; Vallo, C. I. Effect of Different Photoinitiator Systems on Conversion Profiles of a Model Unfilled Light-Cured Resin. Dent. Mater. 2007, 23 (10), 1313-1321.

(20) Corrigan, N.; Shanmugam, S.; Xu, J.; Boyer, C. Photocatalysis in Organic and Polymer Synthesis. Chem. Soc. Rev. 2016, 45 (22), 6165-6212.

(21) Dadashi-Silab, S.; Doran, S.; Yagci, Y. Photoinduced Electron Transfer Reactions for Macromolecular Syntheses. Chem. Rev. 2016, 116 (17), 10212-10275.

(22) Strehmel, B.; Schmitz, C.; Brömme, T.; Halbhuber, A.; Oprych, D.; Gutmann, J. S. Advances of near Infrared Sensitized Radical and Cationic Photopolymerization: From Graphic Industry to Traditional Coatings. J. Photopolym. Sci. Technol. 2016, 29 (1), 111-121.

(23) Bonardi, A. H.; Dumur, F.; Grant, T. M.; Noirbent, G.; Gigmes, D.; Lessard, B. H.; Fouassier, J. P.; Lalevée, J. High Performance Near-Infrared (NIR) Photoinitiating Systems Operating under Low Light Intensity and in the Presence of Oxygen. Macromolecules 2018, 51 (4), 1314-1324.

(24) Bonardi, A.; Bonardi, F.; Noirbent, G.; Dumur, F.; Dietlin, C.; Gigmes, D.; Fouassier, J. P.; Lalevée, J. Different NIR Dye Scaffolds for Polymerization Reactions under NIR Light. Polym. Chem. 2019, 10 (47), 6505-6514.

(25) Shiraishi, A.; Kimura, H.; Oprych, D.; Schmitz, C.; Strehmel, B. Comparison between NIR and UV-Sensitized Radical and Cationic Reactivity of lodonium Salts Comprising Anions with Different Coordination Behavior. J. Photopolym. Sci. Technol. 2017, 30 (6), 633-638.

(26) Schmitz, C.; Halbhuber, A.; Keil, D.; Strehmel, B. NIR-Sensitized Photoinitiated Radical Polymerization and Proton Generation with Cyanines and LED Arrays. Prog. Org. Coatings 2016, 100, 32-46.

(27) Schmitz, C.; Pang, Y.; Gülz, A.; Gläser, M.; Horst, J.; Jäger, M.; Strehmel, B. New High-Power LEDs Open Photochemistry for Near-Infrared- Sensitized Radical and Cationic Photopolymerization. Angew. Chemie 2019, 131 (13), 4445-4450.

(28) Lu, H.; MacK, J.; Yang, Y.; Shen, Z. Structural Modification Strategies for the Rational Design of Red/NIR Region BODIPYs. Chem. Soc. Rev. 2014, 43 (13), 4778-4823.

(29) Loudet, A.; Burgess, K. BODIPY Dyes and Their Derivatives: Syntheses and Spectroscopic Properties. Chem. Rev. 2007, 107 (11), 4891-4932.

(30) L. Thompson, B.; Heiden, Z. Redox Chemistry of BODIPY Dyes. In BODIPY Dyes - A Privilege Molecular Scaffold with Tunable Properties; 2019.

(31) Telitel, S.; Blanchard, N.; Schweizer, S.; Morlet-Savary, F.; Graff, B.; Fouassier, J. P.; Lalevée, J. BODIPY Derivatives and Boranil as New Photoinitiating Systems of Cationic Polymerization Exhibiting a Tunable Absorption in the 400-600 Nm Spectral Range. Polymer. 2013, 54 (8), 2071-2076.

(32) Telitel, S.; Lalevée, J.; Blanchard, N.; Kavalli, T.; Tehfe, M. A.; Schweizer, S.; Morlet-Savary, F.; Graff, B.; Fouassier, J. P. Photopolymerization of Cationic Monomers and Acrylate/Divinylether Blends under Visible Light Using Pyrromethene Dyes. Macromolecules 2012, 45 (17), 6864-6868.

(33) Costa, P.; Sandrin, D.; Scaiano, J. C. Real-Time Fluorescence Imaging of a Heterogeneously Catalysed Suzuki-Miyaura Reaction. Nat. Catal. 2020, 3, 427-437. 
(34) Qiu, H.; Gao, Y.; Boott, C. E.; Gould, O. E. C.; Harniman, R. L.; Miles, M. J.; Webb, S. E. D.; Winnik, M. A.; Manners, I. Uniform Patchy and Hollow Rectangular Platelet Micelles from Crystallizable Polymer Blends. Science. 2016, 352 (6286), 697-701.

(35) Haldar, U.; Lee, H. II. BODIPY-Derived Polymeric Chemosensor Appended with Thiosemicarbazone Units for the Simultaneous Detection and Separation of $\mathrm{Hg}$ (II) lons in Pure Aqueous Media. ACS Appl. Mater. Interfaces 2019, 11 (14), 13685-13693.

(36) Kamkaew, A.; Lim, S. H.; Lee, H. B.; Kiew, L. V.; Chung, L. Y.; Burgess, K. BODIPY Dyes in Photodynamic Therapy. Chem. Soc. Rev. 2013, 42 (1), 77-88.

(37) Miao, X.; Hu, W.; He, T.; Tao, H.; Wang, Q.; Chen, R.; Jin, L.; Zhao, H.; Lu, X.; Fan, Q.; et al. Deciphering the Intersystem Crossing in Near-Infrared BODIPY Photosensitizers for Highly Efficient Photodynamic Therapy. Chem. Sci. 2019, 10 (10), 3096-3102.

(38) Zhao, J.; Xu, K.; Yang, W.; Wang, Z.; Zhong, F. The Triplet Excited State of Bodipy: Formation, Modulation and Application. Chem. Soc. Rev. 2015, 44 (24), 8904-8939.

(39) Koziar, J. C.; Cowan, D. O. Photochemical Heavy-Atom Effects. Acc. Chem. Res. 1977, 11, 334-341.

(40) Allen, M. J.; Page, Z. A. Tracking Photocuring via ATR-FT-IR with Illumination through the ATR Element. Spectrosc. Appl. Noteb. 2020, 35 (2), 78.

(41) Boens, N.; Verbelen, B.; Ortiz, M. J.; Jiao, L.; Dehaen, W. Synthesis of BODIPY Dyes through Postfunctionalization of the Boron Dipyrromethene Core. Coord. Chem. Rev. 2019, 399, 213024.

(42) Wang, X. F.; Yu, S. S.; Wang, C.; Xue, D.; Xiao, J. BODIPY Catalyzed Amide Synthesis Promoted by BHT and Air under Visible Light. Org. Biomol. Chem. 2016, 14 (29), 7028-7037.

(43) Lovell, L. G.; Berchtold, K. A.; Elliott, J. E.; Lu, H.; Bowman, C. N. Understanding the Kinetics and Network Formation of Dimethacrylate Dental Resins. Polym. Adv. Technol. 2001, 12 (6), 335-345.

(44) Thermal Transitions of Homopolymers: Glass Transition \& Melting Point https://www.sigmaaldrich.com/technical-documents/articles/materials-science/polymer-science/thermaltransitions-of-homopolymers.html.

(45) Würth, C.; Grabolle, M.; Pauli, J.; Spieles, M.; Resch-Genger, U. Relative and Absolute Determination of Fluorescence Quantum Yields of Transparent Samples. Nat. Protoc. 2013, 8 (8), 1535-1550.

(46) Polykarpov, A. Y.; Neckers, D. C. Tetramethylammonium Phenyltrialkylborates in the Photoinduced Electron Transfer Reaction with Benzophenone. Generation of Alkyl Radicals and Their Addition to Activated Alkenes. Tetrahedron Lett. 1995, 36 (31), 5483-5486.

(47) Romańczyk, P. P.; Kurek, S. S. Reliable Reduction Potentials of Diaryliodonium Cations and Aryl Radicals in Acetonitrile from High-Level Ab Initio Computations. Electrochim. Acta 2020, 351, 136404.

(48) Romańczyk, P. P.; Kurek, S. S. The Reduction Potential of Diphenyliodonium Polymerisation Photoinitiator Is Not -0.2 V vs. SCE. A Computational Study. Electrochim. Acta 2017, 255, 482-485.

(49) Sabatini, R. P.; McCormick, T. M.; Lazarides, T.; Wilson, K. C.; Eisenberg, R.; McCamant, D. W. Intersystem Crossing in Halogenated Bodipy Chromophores Used for Solar Hydrogen Production. J. Phys. Chem. Lett. 2011, 2 (3), 223-227.

(50) Rachford, A. A.; Ziessel, R.; Bura, T.; Retailleau, P.; Castellano, F. N. Boron Dipyrromethene (Bodipy) Phosphorescence Revealed in [Ir(Ppy) 2(Bpy-C - C-Bodipy)]+. Inorg. Chem. 2010, 49 (8), 3730-3736.

(51) Whited, M. T.; Djurovich, P. I.; Roberts, S. T.; Durrell, A. C.; Schlenker, C. W.; Bradforth, S. E.; Thompson, M. E. Singlet and Triplet Excitation Management in a Bichromophoric Near-Infrared-Phosphorescent BODIPY-Benzoporphyrin Platinum Complex. J. Am. Chem. Soc. 2011, 133 (1), 88-96.

(52) Lee, Y.; Malamakal, R. M.; Chenoweth, D. M.; Anna, J. M. Halogen Bonding Facilitates Intersystem Crossing in lodo-BODIPY Chromophores. J. Phys. Chem. Lett. 2020, 11 (3), 877-884.

(53) Sheng, W.; Wu, Y.; Yu, C.; Bobadova-Parvanova, P.; Hao, E.; Jiao, L. Synthesis, Crystal Structure, and the Deep Near-Infrared Absorption/Emission of Bright AzaBODIPY-Based Organic Fluorophores. Org. Lett. 2018, 20 (9), 2620-2623.

(54) Adarsh, N.; Avirah, R. R.; Ramaiah, D. Tuning Photosensitized Singlet Oxygen Generation Efficiency of Novel Aza-BODIPY Dyes. Org. Lett. 2010, 12 (24), 5720-5723. 
